\title{
EL ASESORAMIENTO FILOSÓFICO EN EL ÁMBITO INTERNACIONAL E IBEROAMERICANO: NOTA HISTORIOGRÁFICA
}

\author{
JOSÉ LUIS ROMERO \\ Universidad Complutense de Madrid \\ joseluisromero@alterconsulta.com
}

RECIBIDO: 15 DE ENERO DE 2010

ACEPTADO: 14 DE ABRIL DE 2010

El objetivo de esta exposición es realizar un repaso esquemático y actualizado de los principales datos y referencias que han nutrido la cada vez más reseñable historia de esta actividad ubicada en el ámbito de la práctica filosófica $\mathrm{y}$ mayormente conocida como asesoramiento $\mathrm{u}$ orientación filosófica. ${ }^{1}$

Abordaremos los hitos más destacados en la historia del asesoramiento filosófico, primero en el ámbito internacional y anglosajón, y posteriormente en el mundo iberoamericano y español.

En ambos casos, estructuraremos la exposición haciendo referencia a: los inicios, las primeras consultas abiertas, las asociaciones, las primeras y principales publicaciones, incluidas las primeras tesis doctorales, los congresos celebrados, los foros virtuales de discusión, las revistas especializadas y los programas de formación.

Con ello, esperamos haber recopilado, actualizado y ofrecido una información de utilidad e interés tanto para el neófito como para el filósofo avezado en esta disciplina.

\footnotetext{
${ }^{1}$ Una versión previa del trabajo que aquí ofrecemos fue presentada como comunicación en el IV Congreso Internacional de la Sociedad Académica de Filosofía, celebrado en febrero de 2009 en la Universidad Complutense de Madrid. Debe también señalarse la deuda que este trabajo tiene con otros análogos anteriormente realizados, como el incluido en Barrientos (2004), a quien agradezco especialmente las precisiones y novedades posteriores que me ha transmitido, y el "Cronograma del asesoramiento filosófico" realizado por Miguel Sancho en Asepraf hasta el año 2007.
} 


\section{Ámbito internacional y anglosajón}

\section{Inicios}

La historia oficial del asesoramiento filosófico marca su inicio en el año 1981, cuando el filósofo Gerd Achenbach abrió su consulta dedicada a esta actividad en Alemania. Sin embargo, el límite real que pueda señalar los inicios resulta mucho más difuso.

Se debate el tipo de actividad desarrollada en 1967 en la consulta del filósofo John van Veen en Holanda, o hacia 1974 por Paul Sharkey como "filósofo residente" en un hospital de Estados Unidos.

Por otro lado, es bien conocido que durante el siglo $\mathrm{XX}$ ha habido notables acercamientos e incluso fusiones por parte de la psicología, en especial aquella de raigambre psicodinámica menos positivista, con la filosofía, en particular con la vertiente fenomenológica y existencial. Sólo así pueden entenderse los desarrollos del psicoanálisis y la psicología existencial y humanista y sus múltiples derivaciones. Cabe también indicar otras aproximaciones, como la de Albert Ellis hacia la filosofía estoica en la naciente psicología cognitiva.

No obstante, nuestro objetivo sería rastrear aquellos acercamientos acontecidos en sentido opuesto, desde la titularidad de la filosofía hacia la práctica terapéutica de la psicología. En este sentido, el psicoanalista Michael Russell atribuye esta naturaleza a su actividad desde los años setenta, si bien no hay duda de que la obra del español Luis Cencillo en esas mismas fechas supone uno de los antecedentes y desarrollos implícitos más sólidos y relevantes a nivel internacional (Pérez et al. 2004, Machado 2009). Pero más allá de estas u otras figuras equipresentes en ambas disciplinas, encontramos la obra del filósofo Peter Koestenbaum, quien también entonces trabajó de manera abierta por una "filosofía clínica" (Koestenbaum 1978).

Ahora bien, el referente explícito más evidente es el del filósofo estadounidense Seymon Hersh, quien en 1980 publica un breve artículo titulado "The counseling Philosopher" (Hersh 1980), donde expone, con terminología y argumentación equivalentes a las actuales, el tipo de 
actividad que conocemos como asesoramiento filosófico, además de presentarse como filósofo "asesor en la práctica privada".

Sin embargo, será en Alemania donde, de la mano del filósofo Gerd Achenbach, el asesoramiento filosófico encontrará su desarrollo más pujante hasta la publicación del primer superventas de Marinoff. Achenbach abre su consulta en 1981, en 1982 crea la Asociación Alemana para la Práctica Filosófica, y en 1984 publica el primer libro de la naciente disciplina (Achenbach 1984).

\section{Consultas}

Tras la inicial consulta de Achenbach en 1981 en Alemania, en 1987 abrirá su consulta Ad Hoogendijk en Holanda y en 1991 hará lo propio Marinoff en Estados Unidos. A partir de aquí, la actividad tendrá representantes en múltiples países de los cinco continentes.

\section{Asociaciones}

Tras la Asociación Alemana creada en 1982, en 1989 se formará la Asociación Holandesa para la Filosofía Práctica ${ }^{2}$ y, de la mano de Shlomit Schuster, el Centro Sophon en Israel, que en 1996 conformará la Sociedad Israeli ${ }^{3}$. Ese mismo año en Inglaterra, pero con clara vocación y proyección internacional, se constituye la Sociedad para la Filosofía en Práctica (SPP) ${ }^{4}$. En 1998 la Sociedad Alemana se transforma en la Sociedad Internacional para la Práctica Filosófica (IGPP) ${ }^{5}$. En ese mismo año surgen la Sociedad Noruega ${ }^{6}$, y también la Asociación Americana (APPA $)^{7}$, liderada por Marinoff, si bien en Estados Unidos ya existía desde 1992 la más plural Sociedad Americana para la Filosofía, el Asesoramiento y la Psicoterapia (ASPCP) ${ }^{8}$. En Italia se han conformado

\footnotetext{
${ }_{3}^{2}$ Vereniging voor Filosofische Praktijk (VFP): http://www.verenigingfilosofischepraktijk.nl.

3 Israeli Society for Philosophical Practice and Counseling (ISPPI): http://sites.google.com/site/thephilosophicalcounselingweb/home.

${ }^{4}$ Society for Philosophy in Practice (SPP): http://www.society-for-philosophy-in-practice.org.

${ }^{5}$ Internationale Gesellschaft für Philosophische Praxis (IGPP): http://www.igpp.org.

${ }^{6}$ Norsk Selskap for Filosofisk Praksis: http://www.nsfp.no.

${ }^{7}$ American Philosophical Practitioners Association (APPA): http://www.appa.edu.

${ }^{8}$ American Society for Philosophy, Counseling \& Psychotherapy (ASPCP): http://www.aspcp.org.
} 
hasta tres asociaciones desde 1999: $\mathrm{SICoF}^{9}, \mathrm{AIP}^{10}$ y Phronesis ${ }^{11}$. También se hay sociedades en Canadá ${ }^{12}$, Suiza ${ }^{13}$ o Sudáfrica ${ }^{14}$.

\section{Publicaciones}

Tras la ya señalada obra pionera de Achenbach (1984), la primera aportación bibliográfica destacable en el ámbito internacional seguramente sea el capítulo que Elliot Cohen escribió sobre "The Philosopher as Counselor" en la primera edición de su Philosophers at work (Cohen 1989). Dos años después, Shlomit Schuster publicó el primer artículo sobre asesoramiento filosófico en una revista académica (Schuster 1991).

En 1995 se publicaron las actas del I Congreso Internacional de Práctica Filosófica sobre asesoramiento filosófico (Lahav y Tillmanns 1995). Más adelante indicaremos los siguientes congresos internacionales, algunas de cuyas actas también han sido publicadas.

En 1999 se produce la publicación de Plato, not Prozac! por parte de Lou Marinoff (Marinoff 1999), y con ella la difusión del asesoramiento filosófico al gran público en las muchas lenguas a las que ha sido traducido.

Ese mismo año publica Schuster su primera obra (Schuster 1999); el año siguiente hará lo propio el canadiense Peter Raabe (Raabe 2000); formuladas un año después, destacamos también la aportación británica de Tim LeBon (LeBon 2001) y la nueva propuesta del israelí Ran Lahav (Lahav 2001).

Señalamos, por último, posteriores obras de Achenbach (2001), Marinoff (2001 y 2003), Raabe (2002 y 2006), Schuster (2003) y Cohen (2007).

\footnotetext{
${ }^{9}$ Società Italiana di Counseling Filosofico (SICoF): http://www.sicof.it.

${ }^{10}$ Associazione Italiana Psicofilosofi (AIP): http://www.psicofilosofia.it.

${ }^{11}$ Phronesis. Associazione Italiana per la Consulenza Filosofica: http://www.phronesis.info.

${ }^{12}$ Canadian Society for Philosophical Practice (CSPP): http://www.philosophicalpractice.ca.

${ }_{14}^{13}$ Netzwerk für praktisches Philosophieren: http://www.philopraxis.ch.

14 Southern African Philosopher Consultants Association: http://www.geocities.com/tjglouw/index.html.
} 
La primera tesis doctoral sobre asesoramiento filosófico fue la realizada por Peter Raabe (1999) en la British Columbia University en la ciudad canadiense de Vancouver.

\section{Congresos}

Hasta el presente se han celebrado nueve congresos internacionales y está programado el décimo para el presente año:

- I Congreso Internacional en Vancouver, Canadá, en 1994.

- II Congreso Internacional en Leusden, Holanda, en 1996.

- III Congreso Internacional en Nueva York, Estados Unidos, en 1997.

- IV Congreso Internacional en Bensberg, Alemania, en 1998.

- V Congreso Internacional en Oxford, Reino Unido, en 1999.

- VI Congreso Internacional en Oslo, Noruega, en 2001.

- VII Congreso Internacional en Copenhague, Dinamarca, en 2004.

- VIII Congreso Internacional en Sevilla, España, en 2006.

- IX Congreso Internacional en Carloforte, Italia, en 2008.

- X Congreso Internacional en Leusden, Holanda, en 2010.

Se han publicado las actas del I Congreso (Lahav y Tillmanns 1995), el V Congreso (Curnow 2001), el VI Congreso (Herrestad et al. 2002) y el VIII Congreso (Barrientos 2006a y 2006b).

\section{Foros}

A finales de 1998 se conformó a nivel internacional y en lengua inglesa el foro de discusión Phil-Counsel ${ }^{15}$.

\section{Revistas}

Las principales revistas especializadas en el campo de la práctica y el asesoramiento filosóficos a nivel internacional son la británica Practical Philosophy ${ }^{16}$, editada por la SPP desde 1998, y la estadounidense

\footnotetext{
${ }^{15}$ Foro de discusión Phil-Counsel: http://groups.yahoo.com/group/phil-counsel.

${ }^{16}$ Practical Philosophy: http://practical-philosophy.org.uk/index.htm.
} 
Philosophical Practice ${ }^{17}$, editada por la APPA desde 2005. Cabe mencionar también la italiana Phronesis ${ }^{18}$, editada por la asociación del mismo nombre.

Otras revistas que deben indicarse, aunque actualmente se encuentran inactivas, son la alemana Zeitschrift fur Philosophische Praxis, anteriormente editada por la IGPP, la estadounidense International Journal of Philosophical Practice ${ }^{19}$, en su momento editada por la ASCPC, y la canadiense Revue de la Pratique Philosophique ${ }^{20}$, a cargo de la también indicada CSPP.

Por último, si bien abarcan el más amplio espectro de la filosofía práctica, también pueden señalarse revistas como la Internacional Journal of Applied Philosophy ${ }^{21}$.

\section{Formación}

Resulta habitual que las distintas asociaciones nacionales elaboren un programa de formación propio. La formación en asesoramiento filosófico apenas tiene espacio hoy en el ámbito universitario, aunque las excepciones son cada vez más numerosas.

En el master de la Facultad de Educación de la Universidad de Haifa, Israel, Ran Lahav ha impartido un breve módulo sobre asesoramiento filosófico de manera intermitente desde 1993. Y desde 2007 el Master Universitario en Filosofías Contemporáneas de la Universidad Estatal de Moldavia ofrece varios cursos destinados a diversas prácticas filosóficas, entre ellos uno centrado en el asesoramiento filosófico.

Italia, sin embargo, resulta pionera en este asunto, pues allí encontramos los primeros masters universitarios centrados en el asesoramiento filosófico. En Venecia, la Università $\mathrm{Ca}^{\prime}$ Foscari Venezia inició la primera edición de su Master Universitario en Asesoramiento Filosófico en el año 2005. En Turín, la Escuela Superior de Formación Rebaudengo, afiliada a la Universidad Pontificia Salesiana de Roma y en

\footnotetext{
${ }^{17}$ Philosophical Practice: http://www.appa.edu/journal.htm.

${ }^{18}$ Phronesis: http://www.phronesis.info/RivistaI.html.

${ }^{19}$ International Journal of Philosophical Practice: http://aspcp.org/ijpp/index.html.

${ }^{20}$ Revue de la Pratique Philosophique: http://www.ustpaul.ca/Philosophy/revue/index.html.

${ }^{21}$ International Journal of Applied Philosophy: http://www.pdenet.org/ijap.html.
} 
colaboración con SICoF y otras instituciones relacionadas con el asesoramiento filosófico, ofrece desde 2009 un Master Universitario en Asesoramiento Filosófico. Y también a partir de esa fecha en la Universidad de Messina, situada en Sicilia, la Facultad de Filosofía y Letras, en colaboración con la asociación Phronesis, oferta un Master Universitario en Asesoramiento y Práctica Filosófica.

\section{Iberoamérica y España}

\section{Inicios}

En el ámbito iberoamericano, seguramente los primeros pasos en el ámbito de práctica filosófica grupal fueron dados entre 1998 y 2000 por Octavio Obando y Carmen Zavala, integrantes de la Asociación Búho Rojo $^{22}$ en Perú, donde surgirá entonces la Sociedad Peruana de Consejería Filosófica y Práctica Filosófica (SOPECPRAFIL) ${ }^{23}$. También hacia 1999 inicia su actividad en consulta privada y cafés filosóficos Roxana Kreimer en Argentina.

\section{Consultas}

En España aparece la primera consulta filosófica en el año 2000, a la par que la traducción del primer libro de Marinoff, cuando abre sus puertas en Barcelona el gabinete filosófico Pharos. Hacia el año 2001 inician su consulta Mónica Cavallé, en Madrid, y algunos miembros del grupo ETOR, como José Ordóñez, Francisco Macera o Francisco Pacheco, en Andalucía. A partir de ahí, serán varios los filósofos asesores que surgirán en diversos puntos del territorio español. En el año 2006 comienza en Madrid el trabajo interdisciplinar e integrador de filosofía y psicología de Alter Consulta.

\footnotetext{
${ }^{22}$ Asociación Búho Rojo: http://www.buhorojo.de.

23 Sociedad Peruana de Consejería Filosófica y Práctica Filosófica (SOPECPRAFIL): http://www.redfilosofica.de/sopecprafil.html.
} 


\section{Asociaciones}

En el año 2002 surge en Madrid la Asociación Española para la Práctica y el Asesoramiento Filosóficos (ASEPRAF) ${ }^{24}$ y en Cataluña la Asociación de Filosofía Práctica de Cataluña (AFPC) ${ }^{25}$. En 1999 se había fundado en Sevilla la Asociación de Estudios Humanísticos y Filosofía Práctica XXI, en cuyo seno surgirá el grupo ETOR (Educación, Tratamiento y Orientación Racional) ${ }^{26}$, enfocado al campo de la orientación filosófica.

En nuestro país vecino, en 2004 aparece la Asociación Portuguesa de Asesoramiento Ético y Filosófico (APAEF) ${ }^{27}$, y en 2008 surgirá la Asociación Portuguesa de Ética y Filosofía Práctica (APEFP) ${ }^{28}$. También han surgido asociaciones en otros países iberoamericanos, como la Sociedad de Filosofía Aplicada de México (SOFIAM) ${ }^{29}$.

\section{Publicaciones}

También en 2002 se publica la primera obra de un autor español sobre el asesoramiento filosófico, que constituye una respuesta crítica por parte de Luis Cencillo (Cencillo 2002) a la obra de Marinoff. De manera paralela, Roxana Kreimer publica la primera obra de esta misma temática en castellano en tierras americanas (Kreimer 2002). Mónica Cavallé, que también entonces publicó una obra de espíritu afín al asesoramiento filosófico (Cavallé 2002), publicará dos años después su posición más perfilada (Cavallé 2004). En esas mismas fechas José Barrientos publica el primer texto que resume la historia y las aportaciones globales de los principales autores del asesoramiento filosófico (Barrientos 2004). Un año después Luis Cencillo presentará su manual más completo (Cencillo 2005), una de las últimas obras de quien ha sido una de las figuras más relevantes del reciente pensamiento español.

\footnotetext{
24 Asociación Española para la Práctica y el Asesoramiento Filosóficos (ASEPRAF): http://www.asepraf.org.

${ }^{25}$ Asociación de Filosofía Práctica de Cataluña (AFPC): http://www.afpc.org.es.

${ }^{26}$ Grupo ETOR (Educación, Tratamiento y Orientación Racional): http://www.grupoetor.org.

${ }^{27}$ Associação Portuguesa de Aconselhamento Ético e Filosófico (APAEF): http://www.apaef.com.

${ }^{28}$ Associação Portuguesa de Ética y Filosofia Práctica (APEFP): http://apefp.blogspot.com.

${ }^{29}$ Sociedad de Filosofía Aplicada de México (SOFIAM): http://sofiam.org.
} 
Destacamos después la obra coordinada por Mónica Cavallé y Julián Domingo Machado, en la que también han colaborado Roxana Kreimer, Luis Cencillo y José Luis Romero (Cavallé y Domingo 2007), así como las obras coordinadas por José Barrientos sobre la relación con la práctica y orientación filosófica de figuras ilustres de la historia de la filosofía (Barrientos 2006c) y de la historia de la filosofía española (Barrientos 2009b), y sobre la relación entre la filosofía aplicada y el mundo académico (Barrientos 2010). Otras obras de autoría hispana en este ámbito han sido las de Ortega (2003), Altisen (2003), Guzmán (2007), Puigferrat (2008), Arnaiz et al. (2008), Barrientos y Ordóñez (2008) y Barrientos y Dias (2009).

La primera tesis doctoral en español, y también la primera presentada en Europa sobre esta temática, ha sido realizada por Barrientos (2009a) en la Universidad de Sevilla.

\section{Congresos}

En 2004 se celebró en la Universidad de Sevilla el "I Congreso Iberoamericano y de habla hispana para el asesoramiento y la orientación filosófica", y en ese mismo recinto tuvo lugar en 2006 el ya indicado VIII Congreso Internacional de Práctica Filosófica. Las actas de ambos eventos fueron publicadas posteriormente (Barrientos et al. $2005 \mathrm{y}$ Barrientos 2006a y 2006b).

Por otra parte, son ya notables las jornadas y seminarios formales que sobre la temática de la práctica filosófica y la filosofía aplicada se han desarrollado en diversas ciudades españolas, como las organizadas por el grupo ETOR en la Universidad de Sevilla sobre filosofía y medicina, o las organizadas por Victoria Caro en el Ateneo de Madrid y varias universidades madrileñas sobre filosofía práctica y musicoterapia.

\section{Foros}

Desde 2003 y 2004 funcionan respectivamente los foros de discusión en lengua castellana de ASEPRAF $^{30}$ y FIACOF (Foro Internacional para el

\footnotetext{
${ }^{30}$ Foro de discusión de ASEPRAF: http://es.groups.yahoo.com/group/asepraf.
} 
Asesoramiento, la Consultoría y la Orientación Filosófica, coordinado por José Barrientos) $)^{31}$.

\section{Revistas}

La revista especializada más relevante que ha surgido en lengua castellana ha sido la revista del grupo ETOR, apoyada por la Facultad de Filosofía de la Universidad de Sevilla, de la cual se publicaron cuatro números entre los años 2003 y 2005. En el año 2010, desde esa misma universidad y con periodicidad anual, toma el relevo la presente revista Haser. Revista internacional de filosofía aplicada y orientación filosófica ${ }^{32}$.

\section{Formación}

Respecto a la formación, el primer programa formativo ofertado en el territorio español es el Curso de Formación y Entrenamiento de Filósofos Asesores realizado por ASEPRAF; es el único centrado en el asesoramiento filosófico y, desde el año 2003 hasta la actualidad, ha tenido cuatro ediciones.

No obstante, sobre el ámbito general de la práctica filosófica, ha habido dos universidades españolas que han ofertado programas de postgrado como títulos propios, si bien hoy sólo está vigente uno de ellos. La Universidad de Sevilla, donde se ha constituido formalmente un grupo de investigación en filosofía aplicada y se han realizado diversos seminarios de investigación en este ámbito, así como también varias jornadas ya indicadas sobre filosofía y medicina, ofreció la primera edición del título de Experto Universitario en Filosofía Aplicada a la Orientación Filosófica en el curso 2006/2007, y en este año 2010 ofrece su primera edición del Máster en Filosofía Aplicada.

\footnotetext{
${ }^{31}$ Foro de discusión FIACOF: http://es.groups.yahoo.com/group/FIACOF.

${ }^{32}$ Haser. Revista internacional de filosofía aplicada y orientación filosófica. Se prevé que se cuelgue en el repositorio de revistas científicas de DIALNET.
} 
Por su parte, la Universidad de Barcelona presentó su primera edición del Máster en Práctica Filosófica y Gestión Social en el curso 2007/2008, pero la posterior oferta ha sido clausurada hasta el momento actual.

Fuera del territorio español pero dentro del ámbito iberoamericano, también la Universidad Vasco de Quiroga, en la ciudad mexicana de Morelia, ofrece varios cursos de postgrado sobre filosofía aplicada desde el año 2009, con perspectivas de conversión a máster en el presente año.

\section{Bibliografía}

Achenbach, G.B. (1984). Philosophische Praxis. Köln: Jürgen Dinter. Achenbach, G.B. (2001). Lebenskönnerschaft. Freiburg: Herder. Altisen, C. (2003). Asesoría filosófica y mediación. Buenos Aires: Libros en Red. (www.librosenred.com)

Arnaiz, G. et al. (2008). Saber pensar para saber vivir. Filosofía aplicada, identidad y sufrimiento. Sevilla: Fénix.

Barrientos, J. (2004/2005). Introducción al asesoramiento y la orientación filosófica. Sevilla: Kronos/X-XI, 2004 (reed: Las Palmas: Idea, 2005).

Barrientos, J. et al. (eds.) (2005). La filosofía a las puertas del tercer milenio. I Congreso iberoamericano y de habla hispana para el asesoramiento y la orientación filosófica. Sevilla: Fénix.

Barrientos, J. (ed.) (2006a). Dominios de aplicación práctica de la filosofía. Sevilla: Ediciones X-XI.

Barrientos, J. (ed.) (2006b). Philosophical Practice: from theory to practice. Sevilla: Ediciones X-XI.

Barrientos, J. (ed.) (2006c). Entre historia y orientación filosófica. La orientación filosófica en la historia del pensamiento. Vol. I y II. Sevilla: Ediciones X-XI.

Barrientos, J. (2009a). Vectores zambranianos para una teoría de la filosofía aplicada a la persona. Tesis doctoral. Sevilla: Universidad de Sevilla.

Barrientos, J. (ed.) (2009b). Filosofía aplicada y circunstancia española. Sevilla: Ceshvaq. 
Barrientos, J. (ed.) (2010). Filosofía aplicada y universidad. Madrid: Visión.

Barrientos, J. y Dias, J. (2009). ¿Felicidad o conocimiento? La filosofía aplicada como búsqueda de la felicidad y el conocimiento. Sevilla: Project.

Barrientos, J. y Ordóñez, J. (eds.) (2008). Filosofía aplicada a personas y grupos. Sevilla: Doss.

Cavallé, M. (2002). La sabiduría recobrada. Filosofía como terapia. Madrid: Oberón.

Cavallé, M. (2004). La filosofía, maestra de vida. Respuestas a las inquietudes de la mujer de hoy. Madrid: Aguilar.

Cavallé, M. y Machado, J.D. (eds.) (2007). Arte de vivir, arte de pensar. Iniciación al asesoramiento filosófico. Bilbao: Desclée de Brouwer.

Cencillo, L. (2002). Cómo Platón se vuelve terapeuta. Madrid: Syntagma.

Cencillo, L. (2005). Asesoramiento: qué técnicas, qué filosofías. Las Palmas: Idea.

Cohen, E.D. (ed.) (1989/2000). Philosophers at Work: an Introduction to the Issues and Practical Uses of Philosophy. NY: Holt, Rinehart and Winston. $\left(2^{\text {nd }}\right.$ Edition: Philosophers at Work: Issues and Practice of Philosophy. Orlando: Harcourt Brace, 2000).

Cohen, E.D. (2007). The New Rational Therapy. New York: Rowman \& Littlefield Publishers.

Curnow, T. (ed.) (2001). Thinking Through Dialogue. Oxted: Practical Philosophy Press.

Guzmán, R. (2007). La mujer serena. Pensamiento, filosofía y mundo femenino. Barcelona: Sirpus.

Herrestad, H. et al. (eds.) (2002). Philosophy in Society. Oslo: Unipub.

Hersh, S. (1980). "The Counseling Philosopher". The Humanist, 40(3): 32-34.

Koestenbaum, P. (1978). The new image of the person. The theory and practice of clinical philosophy. Westport, Conn: Greenwood Press.

Kreimer, R. (2002/2005). Artes del buen vivir: filosofía para la vida cotidiana. Buenos Aires: Anarres, 2002 (reed: Filosofía para la vida cotidiana. Las Palmas: Idea, 2005). 
Lahav, R. (2001). "Philosophical Counselling as a Quest for Wisdom". Practical Philosophy, 4(1): 6-18.

Lahav, R. y Tillmanns, M. (eds.) (1995). Essays on Philosophical Counseling. NY: University Press of America.

LeBon, T. (2001). Wise Therapy: Philosophy for Counsellors. London: Continuum.

Machado, J.D. (2009). "Luis Cencillo y el asesoramiento filosófico". Paideia. Revista de filosofía y didáctica filosófica, 84: 117-136.

Marinoff, L. (1999/2000). Plato, not Prozac! Applying Philosophy to Everday Problems. NY: Harper-Collins, 1999 (trad. cast: Más Platón y menos prozac. Filosofía para la vida cotidiana. Barcelona: Ediciones B, 2000).

Marinoff, L. (2001). Philosophical Practice. NY: Academic Press.

Marinoff, L. (2003/2004). The Big Questions. How Philosophy Can Change Your Life. NY: Bloomsbory, 2003 (reed: Therapy for the Sane. 2004) (trad. cast: Pregúntale a Platón. Cómo la filosofía puede cambiar tu vida. Barcelona: Ediciones B, 2004).

Ortega, P. (2003). Curar con el pensamiento. Madrid: Laberinto.

Pérez, C. et al. (2004). "Orígenes de la terapia filosófica en España: Luis Cencillo". Ponencia presentada en el I Congreso Iberoamericano para el Asesoramiento y la Orientación Filosófica. Sevilla, 14-16 de abril de 2004.

Puigferrat, J. (2008). El pez que vivía fuera del agua. Cómo la filosofía práctica puede ayudar a encaminar nuestra vida. Madrid: Maeva.

Raabe, P.B. (1999). Philosophy of Philosophical Counseling. PhD Thesis. Vancouver: University of British Columbia.

Raabe, P.B. (2001). Philosophical Counseling: Theory and Practice. Westport, Conn: Praeger.

Raabe, P.B. (2002). Issues in Philosophical Counseling. London: Praeger.

Raabe, P.B. (ed.) (2006). Philosophical Counselling and the Unconscious. New York: Trivium Publications.

Schuster, S.C. (1991). "Philosophical Counselling". Journal of Applied Philosophy, 8(2): 219-223. 
Schuster, S.C. (1999). Philosophy Practice: An Alternative to Counseling and Psychotherapy. Westport, Conn: Praeger.

Schuster, S.C. (2003). The Philosopher's Autobiography. Westport, Conn: Praeger. 